\title{
A Study of Clinical-Radiological, Pathological Profile and Treatment Outcome of Patients Admitted to a Tertiary Care Center with Haemoptysis
} Adrian P Noriega Aldave*

Virginia Tech Carilion School of Medicine, Roanoke, USA

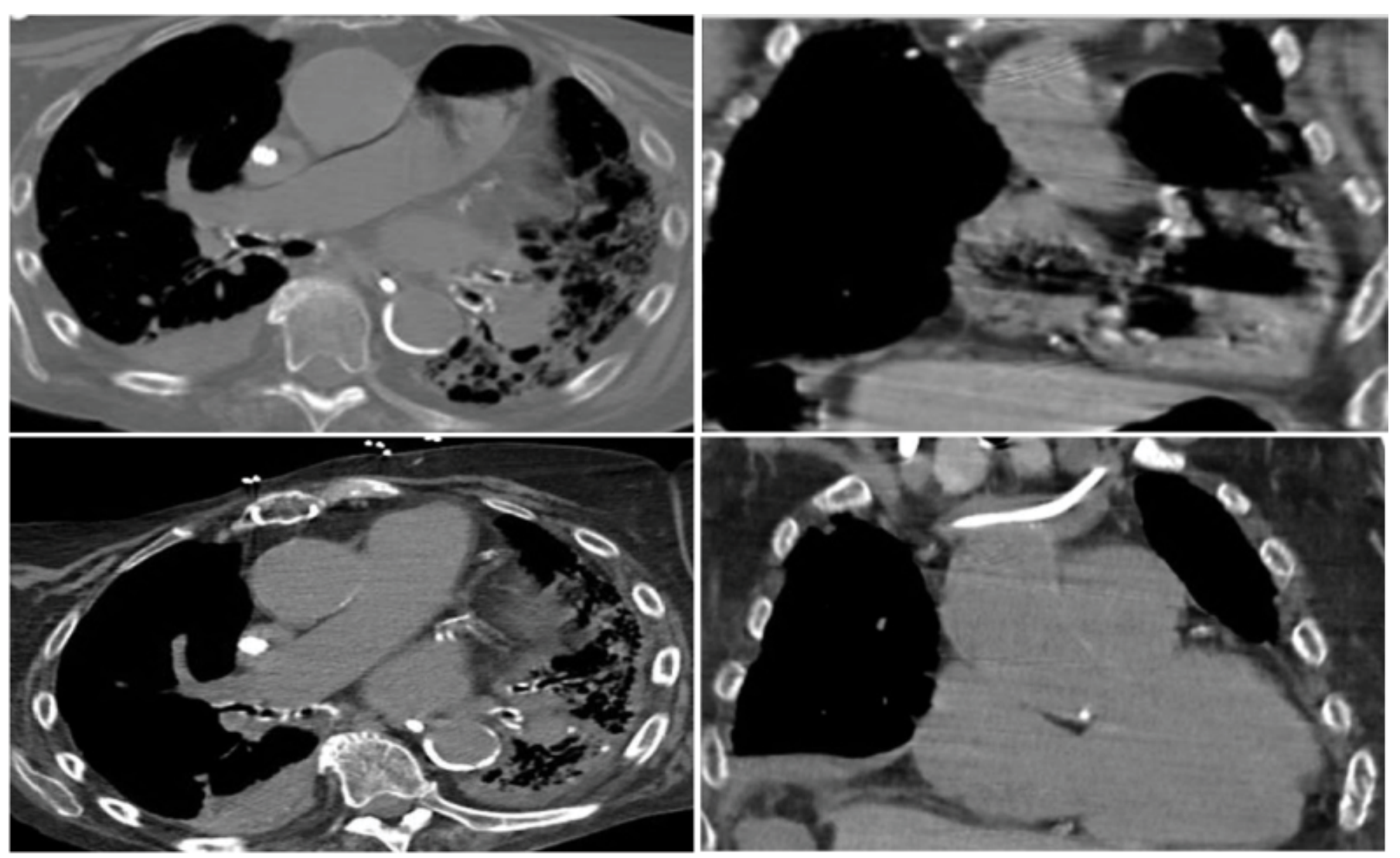

Figure 1: CT chest showing venous air embolism in the upper images and the resolution after 24 hours.

\section{Clinical Image}

Venous air embolism (VAE) is a life threatening condition usually related to iatrogenia causing entrance of atmospheric gas into the venous system. It has been reported in venous cannulation, insufflation of gas into a body cavity, and hemodialysis. We present a case of massive VAE following hemodialysis.

A 77-year-old woman with atrial fibrillation, chronic obstructive lung disease, chronic kidney disease, diabetes and hypertension was admitted with sepsis secondary to aspiration pneumonia. Subsequently she developed worsening renal failure requiring hemodialysis through her internal jugular vein. During a hemodialysis session, she developed profound hypotension requiring dialysis termination, and immediately thereafter becoming more hypoxemic. A non-contrast chest CT was ordered and revealed a large amount of air in the main pulmonary artery, right atrium and right ventricle (Figure 1).

The patient was immediately positioned in left lateral decubitus, trendelenburg position and $100 \%$ oxygen was provided with a nonrebreather mask. Over the next 24 hours her hemodynamics and oxygenation improved. A non-contrast chest CT 72 hours later showed complete resolution of the VAE.
While there is an increasing frequency of dialysis performed through temporary catheters, very few cases of VAE have been reported. Although improvement in equipment may explain its low incidence, it is also possible that there is under-recognition of this complication. Patients could have transient periods of hypotension that may be attributed to other factors. Additionally, as we see an aging and more complicated population, hypotension and also other associated side effects including respiratory distress, may not be unusual during hemodialysis, particularly in acute ill patients. Therefore, recognition of air embolism as a cause of these complications is important and requires a heightened level of awareness by the entire team.

*Corresponding author: Adrian P Noriega Aldave, Virginia Tech Carilion Schoo of Medicine, Roanoke, USA, Tel: 13342331417; E-mail: adnoriegaa@gmail.com

Received December 13, 2017; Accepted December 19, 2017; Published December 26, 2017

Citation: Noriega Aldave AP (2017) Massive Venous Air Embolism in a Patient with Haemodialysis. J Pulm Respir Med 7: 438. doi: 10.4172/2161-105X.1000438

Copyright: () 2017 Noriega Aldave AP. This is an open-access article distributed under the terms of the Creative Commons Attribution License, which permits unrestricted use, distribution, and reproduction in any medium, provided the original author and source are credited. 